\title{
Electrical Conductivity Modeling of Polypropylene Composites Filled with Carbon Black and Acetylene Black
}

\author{
Abdelhafid Merzouki and Naceredine Haddaoui \\ Laboratoire de Physico-Chimie des Hauts Polymères (LPCHP), Université Ferhat Abbas, Sétif, Algeria \\ Correspondence should be addressed to Abdelhafid Merzouki, hafid_merzouki@yahoo.fr \\ Received 13 February 2012; Accepted 7 March 2012 \\ Academic Editors: A. Uygun and J. I. Velasco \\ Copyright (c) 2012 A. Merzouki and N. Haddaoui. This is an open access article distributed under the Creative Commons \\ Attribution License, which permits unrestricted use, distribution, and reproduction in any medium, provided the original work is \\ properly cited. \\ Composites of polypropylene filled with carbon black or acetylene black at different concentrations were prepared by melt mixing \\ followed by compression molding. The influences of filler type and filler concentration on the composites conductivity were \\ studied. It was found that the percolation threshold is located at a lower concentration in composites filled with the acetylene \\ black, than that of the composites filled with carbon black. The model of Mamunya gives a fairly good agreement in the evaluation \\ of the conductivity of polymeric composites loaded with carbon black or acetylene black, beyond the percolation threshold. \\ The Boltzman equation was adopted to develop a model that represents more faithfully all results obtained. The expressions \\ of the electrical conductivity, calculated with the model developed, are in good agreement with experimental results for the entire \\ concentration range studied in linear or semilogarithmic scale.
}

\section{Introduction}

We have previously discussed the interest of conductive composites and the modern technology requirement for these products. To better control the applications of these materials, it is necessary to develop fundamental knowledge about the factors that control the conductivity in composites. These can then be used to find and develop new composites used for new fields [1-5].

Specifically, the mechanisms that control the conductivity will help selecting components in advance for a composite with specific conductivity and will save time and money.

Usually the electrical conductivity of composites depends on the volume fraction of the conductive filler. When the filler concentration is increased in the composite, the filler particles develop points of contact between them. When these points become sufficiently numerous, they may form a continuous network within the matrix for the transport of conduction electrons. The conductivity of the composites can be interpreted using the theories of percolation [6-12].

There are several factors that may have a significant effect on the value of the percolation threshold and the level of maximum conductivity. For instance, particles or fibers improve the electrical transport. Theoretical models taking into account these factors have been proposed to predict the behaviour of the electrical conductivity of composites. Most models are based on the volume fraction of the filler, while other factors can affect the conductivity of the composite. The factors neglected by the models can affect the rate at which percolation occurs. These are factors related to the nature of the filler or the nature of the matrices. Others are related to the preparation technique and operating conditions.

Among the models that predict the conductivity of the materials according to the interactions between the polymer and the filler, particularly those involving the surface tension and surface energy components are those proposed by Mamunya et al. [13, 14] and Clingerman et al. [15].

Mamunya and coworkers have studied the conductivity of composites based on the filler concentration in different polymers to evaluate the influence of polymer-filler interactions on the conductivity.

The proposed model takes into account the surface energy of the polymer as well as the filler and the aspect ratio of 
the filler. Beyond the percolation threshold, the conductivity of the polymer-carbon black composite can be predicted using Mamunya equation [16]:

$$
\log \sigma_{m}=\log \sigma_{c}+\left(\log \sigma_{F}-\log \sigma_{c}\right)\left(\frac{\phi-\phi_{c}}{F-\phi_{c}}\right)^{k},
$$

where $k$ is a constant, which depends upon the filler fraction, percolation threshold, and the interfacial tension.

$\sigma_{m}$ : composite conductivity.

$\sigma_{c}$ : composite conductivity at the percolation threshold.

$\sigma_{F}$ : composite conductivity at the maximum packing fraction.

$F$ : the maximum packing fraction $(30 \mathrm{~V} \%)$.

$\phi$ : volume fraction.

$\phi_{c}$ : percolation threshold.

Mamunya model offers a good agreement between calculated and experimental results for carbon black in different polymers. However, it is not valid for other types of fillers. New models were introduced to make the theoretical models closer to the experimental results $[15,17-25]$.

\section{Experimental}

\subsection{Materials}

2.1.1. Polymer. The polymer used in this study is polypropylene (PP) (trade name ISPLEN), manufactured by Quimica Repsol (Spain).

2.1.2. The Fillers. The fillers used are carbon black and acetylene black.

(i) Carbon black (CB) is a furnace Black type and is a conductor grade initially intended for use as an electrolyte paste in dry cells. The $\mathrm{CB}$ has a density of $1.8\left(\mathrm{gr} / \mathrm{cm}^{3}\right)$ and a specific area of $42 \mathrm{~m}^{2} / \mathrm{gr}$ and is manufactured by Ensagri (Belgium) under the trade name: Super S Battery Black.

(ii) Acetylene black $(A B)$ is a conductor grade with a great purity. The AB has a density of $1.8\left(\mathrm{gr} / \mathrm{cm}^{3}\right)$ and a specific area of $110 \mathrm{~m}^{2} / \mathrm{gr}$. It is manufactured by ElfAtochem (France) under the trade name: "YS".

2.2. Sample Preparation. Polymer/carbon black mixtures were prepared in a Schwabenthan Polymix 80T two roll mill (speed ratio 1/1.2), by gradually adding the appropriate amount of the filler in the polymer matrix.

The matrix/filler mixing was performed at a temperature slightly above the melting or softening point of the polymers. The simplicity of this technique allows the preparation of uniform and homogeneous samples in terms of filler distribution in the matrix. It can adapt to any form of the polymer, whether it is granulates, powder, or fibers.

Once the filler was entirely added, after 10 minutes of mixing, the resulting composites were removed from the roll mill in a form of slabs or small crumbs.

In the case of the PVC-based composite, a stabilized semirigid formulation was homogenized and premixed in a propeller mixer before being mixed again in the roll mill. All filler concentrations in this study are expressed in volume percent.

The slabs obtained from the roll mill were then grinded and were molded by compression molding to prepare different specimen for testing. The compression molding was carried out using a DAVENPORT 25 T hydraulic press. After preheating at the mixing temperature of the particular polymer for 2 minutes, and degassing 2 or 3 times, the material was pressed under a pressure of 150 bars for 5 minutes. At the end of this cycle, the specimen plates were removed from the press and immediately immersed in water.

2.3. Characterization. The volumetric conductivity was measured according to the J. C. Dubois method $[25,26]$ on specimen having dimensions of $20 \times 10 \times 4 \mathrm{~mm}^{3}$. The setup used was composed of an electrical generator (PHILIPS $P E$ 1536, DC Power supply 20V-2A), a variable protection resistance (AOIP), a voltmeter (PRACITRONIC mv 21), and an amperemeter (PHYWE). The transversal resistance (R) across the specimen was measured between two cupper electrodes on both sides and then was converted to volumetric resistivity $\rho$, " $\rho=(R \cdot l \cdot w) / t$ ", where $l, w$, and $t$ are the specimen length, width, and thickness, respectively. The electrical conductivity $(\sigma)$ is the inverse of the resistivity $(\sigma=1 / \rho)$.

\section{Results and Discussion}

3.1. Experimental Results. The conductivity of the polypropylene composites filled with carbon black or acetylene black are shown in Figure 1. It shows the continuous increase of the conductivity of the polymer as a function of filler concentration. At low filler loading, the composites have a negligible conductivity corresponding to the usual value of pure polypropylene $\left(10^{-14}-10^{-16} \mathrm{~S} \cdot \mathrm{m}^{-1}\right)$. At high filler concentrations, the materials exhibit a conductivity of about $10 \mathrm{~S} \cdot \mathrm{m}^{-1}$

In the case of acetylene black, the onset of the percolation threshold is located at a concentration of approximately $P_{1} \approx$ $2.6 \%$ while that of carbon black is around $P_{2} \approx 5.3 \%$ by volume (Figure 1). These values were obtained by amplifying the scale $(0-8 \mathrm{~V} \%)$ in order to locate the percolation threshold.

The shape of the curves is similar for both types of fillers. The difference is in the location of the percolation threshold and the divergence in the materials conductivity beyond the percolation threshold. In this context, the PP/AB composite becomes more conductive than PP/CB composite. With the acetylene black, the percolation threshold is located at a lower concentration, than that of carbon black. Beyond the percolation threshold, at equal filler concentration the acetylene black composites have a higher conductivity.

Thus for a given loading rate of $20 \%$ by volume, the conductivity of composites loaded by the $\mathrm{AB}$ is equal to $7.5 \mathrm{~S} \cdot \mathrm{m}^{-1}$, whereas it is equal to $4 \mathrm{~S} \cdot \mathrm{m}^{-1}$ for composites loaded by the CB. This information is most obvious on the representation on a logarithmic scale. 


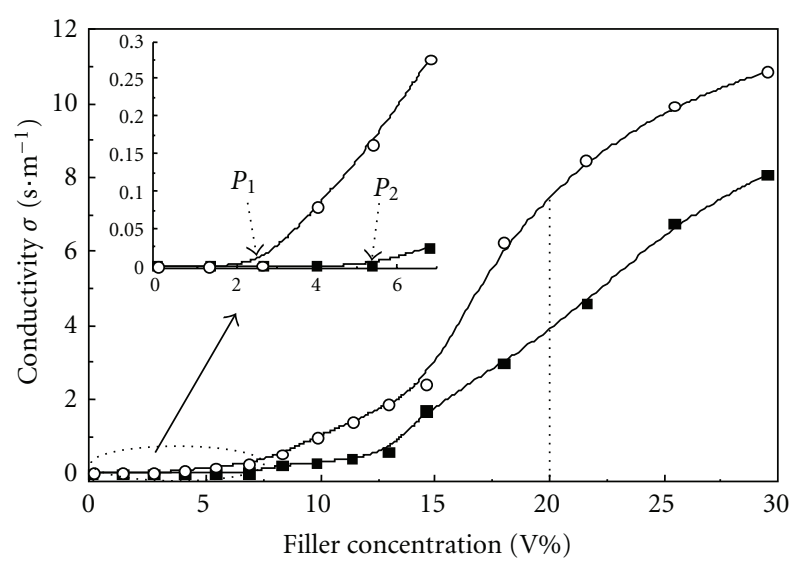

$\rightarrow-\mathrm{PP} / \mathrm{CB}$

$-\mathrm{PP} / \mathrm{AB}$

FIGURE 1: Variation of the conductivity as a function of carbon black $(\mathrm{CB})$ and acetylene black $(\mathrm{AB})$ concentration in the polypropylenebased composites.

This difference in behaviour discrepancy can be attributed to the dispersion mode of the fillers due to the affinity between the polymer matrix and the two fillers. Surface tension is a parameter that can explain this discrepancy, carbon black has very high polar surface tension, which increases with its specific surface area, $\gamma_{c} \geq 70 \mathrm{mj} / \mathrm{m}^{2}$ and can reach several hundred $\mathrm{mJ} / \mathrm{m}^{2}$ of the carbon black having a high specific surface area.

The polymers that are relatively compatible with carbon black are those which are polar such as PVC whose surface tension is $40 \mathrm{~mJ} / \mathrm{m}^{2}$. Nonpolar polymers such as polypropylene whose surface tension is $\approx 30 \mathrm{~mJ} / \mathrm{m}^{2}$ are less compatible with carbon black.

The surface area of the acetylene black used $\left(110 \mathrm{~m}^{2} / \mathrm{gr}\right)$ is almost three times that of the carbon black $\left(42 \mathrm{~m}^{2} / \mathrm{gr}\right)$. Therefore, the acetylene black, which has a surface tension greater than that of carbon black is, less compatible with the PP matrix and tends to disperse in the form of agglomerates which is the most advantageous dispersion mode for an improved conduction of electricity. The carbon black tends to disperse in the form of larger aggregates in smaller numbers isolated from each other within the polymer matrix with fewer points of contact to form a conductive network.

The semilogarithmic plot of the variation of conductivity with the filler concentration seems to be more representative and the transitions in the filler distribution in the PP matrix are more explicit. The location of the percolation threshold is more pronounced and more accurate on the log $(\sigma)=f(c)$ curves. The onset of percolation is located at $I_{1}=1.5$ for $\mathrm{PP} / \mathrm{AB}$ composites and $I_{2}=4 \mathrm{~V} \%$ for $\mathrm{PP} / \mathrm{CB}$ composites. The critical concentrations $\left(I_{1}^{\prime}, I_{2}^{\prime}\right)$ are located at $4 \%$ and $7.5 \%$ volume fraction for acetylene black and carbon black respectively. These concentrations correspond to the formation of a continuous network of filler particles being in an intimate contact inside the composites (Figure 2).

The area corresponding to the percolation threshold is characterised by a drastic increase of the composite

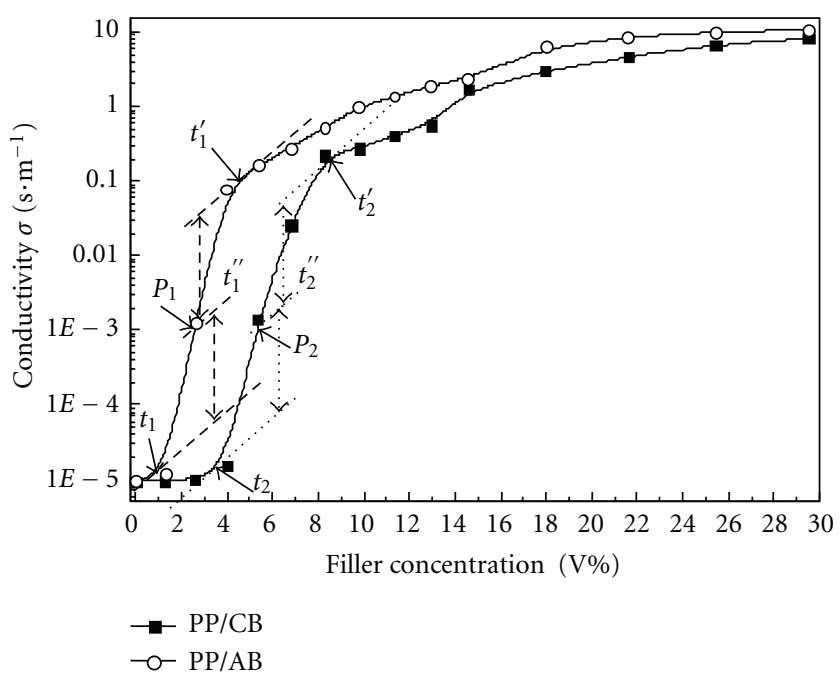

FIGURE 2: Semilogarithmic plot of the variation of the conductivity as a function of carbon black and acetylene black concentration in a polypropylene-based composites.

conductivity for a low filler loading. For a change in the filler concentration from $I_{1}=1.5$ to $I_{2}=4$ of $\mathrm{AB}$, and from $I_{1}^{\prime}=4$ to $I_{2}^{\prime}=7.5 \mathrm{~V} \%$ of $\mathrm{CB}$, the conductivity increases sharply from $10^{-5} \mathrm{~S} \cdot \mathrm{m}^{-1}$ to $0.1 \mathrm{~S} \cdot \mathrm{m}^{-1}$. Above this range the conductivity increases moderately and tends to reach the conductivity of the filler which is $10 \mathrm{~S} \cdot \mathrm{m}^{-1}$.

To determine the percolation threshold graphically which is at or close to the inflection point in the semi- logarithmic curves (Figure 3), we have drawn two parallel tangents $\left(t_{1}^{\prime \prime}\right.$ and $\left.t_{2}^{\prime \prime}\right)$, which pass through the characteristic points $\left(I_{1}\right.$ and $\left.I_{1}^{\prime}\right)$ and $\left(I_{2}\right.$ and $\left.I_{2}^{\prime}\right)$ of each curve. The inflection point is the intersection of the curve with the parallel line with respect to the first two tangents, which runs right in the middle of them. Hence it was found that the inflection point is located at $2.6 \%$ for the acetylene black and at 5.3 volume $\%$ for the carbon black.

The values found are very close to the percolation threshold values that we estimated by amplification of the scale of the linear curves in Figures 1 and 2.

The curves seem to follow the general trend noted in the literature. At low filler loading, the conductivity increases slowly. But at the onset of the percolation threshold the conductivity increases drastically as illustrated in Figure 2 on a semilogarithmic scale. Beyond a certain critical concentration this increase seems to be evolving more moderately [2124].

3.2. Modeling Results. Several existing models were tested to find those that best describe the behavior of the composites studied. Among the models tested, that of Mamunya [1315] seems to be more appropriate. It takes into account the parameters previously neglected by other researchers. It gives a good agreement in the case of polymer composites filled with carbon black beyond the percolation threshold $[14,15,17-22]$. Mamunya model cannot be used for filler concentrations lower than those of the percolation threshold and the term $((x-2,61) / 27,39)$ becomes negative in this 


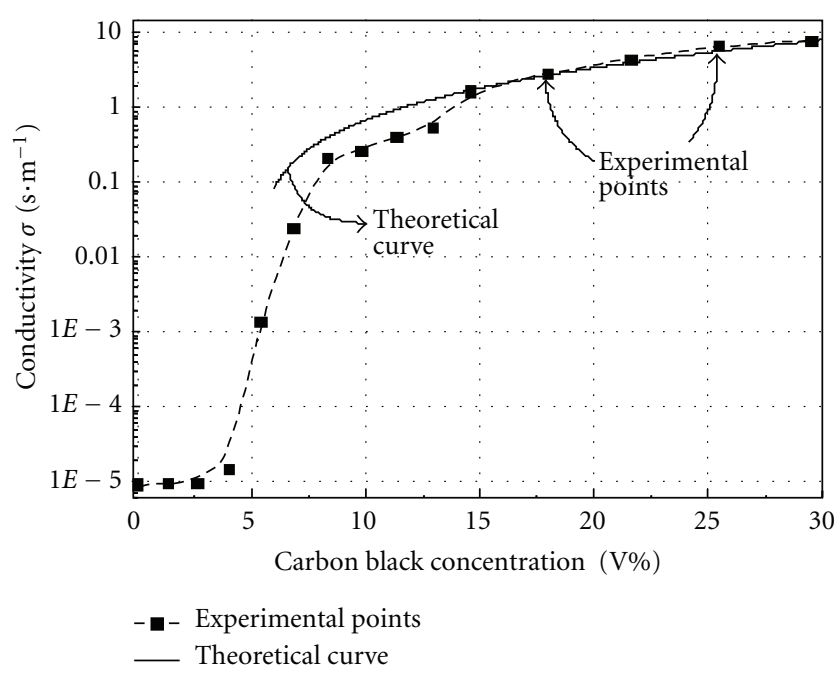

Figure 3: Semilogarithmic plot of the variation of the theoretical (Mamunya model) and the experimental conductivity as a function of carbon black concentration in the polypropylene-based composites.

area. Figures 3 and 4 show the comparison between the experimental points and theoretical points.

In Figure 3, which shows the evolution of the composites conductivity as a function of carbon black concentration, there is a fairly good superposition between the theoretical values and experimental values, especially for high carbon black concentrations as shown in Figure 4, which shows the evolution of the conductivity of composites as a function of acetylene black concentration; there is a difference between the theoretical and the experimental values. This is explained by the fact that Mamunya model was designed primarily to predict the behavior of polymers filled with carbon black beyond the percolation threshold [14, 15, 17-22].

In the representation of Mamunya model with a linear scale, the agreement of the experimental values with the theoretical ones is less obvious. If in the case of $\mathrm{PP} / \mathrm{CB}$ composites the theoretical and experimental values are relatively close, a maximum uncertainty of $15 \%$ is noted for the filler concentration of $25 \mathrm{~V} \%$. With acetylene black, the difference is more important; it reaches the $25 \%$ uncertainty at a filler concentration of $25 \mathrm{~V} \%$ (Figures 5 and 6).

This discrepancy in the prediction of the conductivity with the two filler highlights the existence of interactions that differ from one filler to another and from one matrix to another. The theoretical values of the parameters obtained using Mamunya model are given in Table 1 . By applying the values found:

(i) Mamunya equation model applied to $\mathrm{PP} / \mathrm{CB}$ composites:

$$
\begin{aligned}
\log \left(\sigma_{m}\right)= & \log (0.014) \\
& +[\log (8.1)-\log (0.014)]\left(\frac{\phi-5.3}{30-5.3}\right)^{0.2} .
\end{aligned}
$$

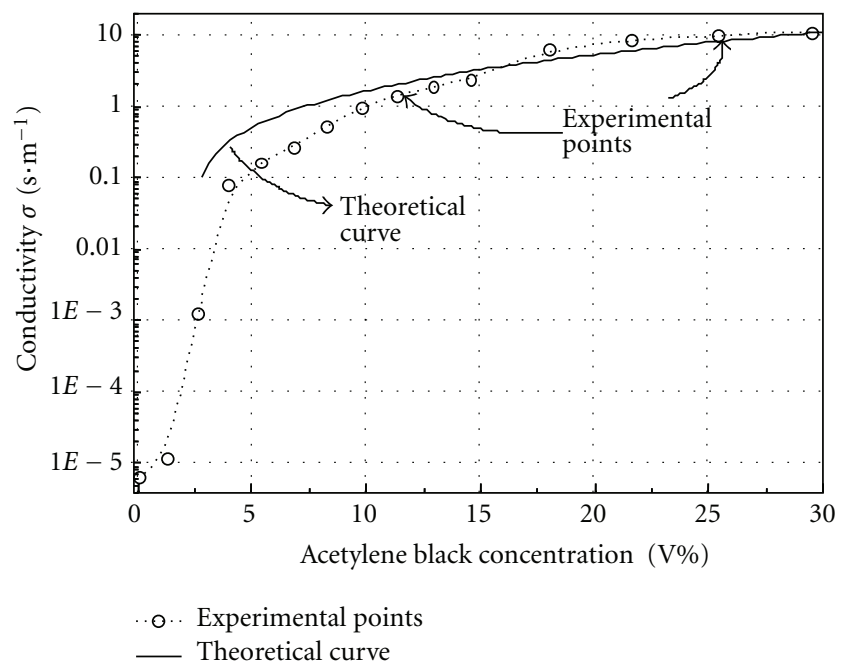

FIGURE 4: Semilogarithmic plot of the variation of the theoretical (Mamunya model) and the experimental conductivity as a function of acetylene black concentration in the polypropylene-based composites.

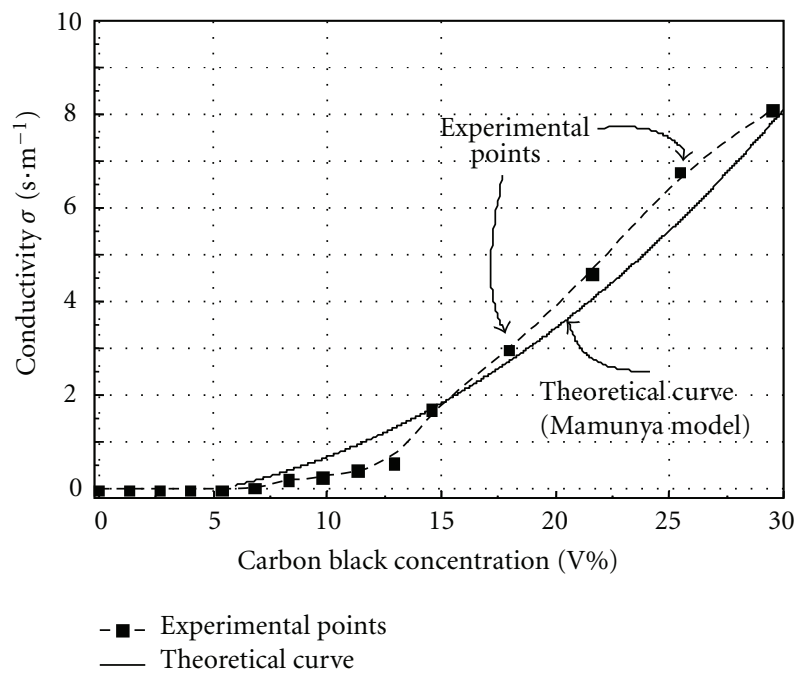

FIGURE 5: Variation of the theoretical (Mamunya model) and the experimental conductivity as a function of carbon black concentration in the polypropylene-based composites.

(ii) Mamunya equation model applied to $\mathrm{PP} / \mathrm{AB}$ composites:

$$
\begin{aligned}
\log \left(\sigma_{m}\right)= & \log (0.0127) \\
& +[\log (10.89)-\log (0.0127)]\left(\frac{\phi-2.6}{30-2.61}\right)^{0.25} .
\end{aligned}
$$

We used different mixing laws to describe the electrical conductivity of the samples. The use of these laws, in their original forms, did not give a satisfactory agreement with the experimental results. This may be due to the fact that several parameters such as the matrix nature, particle type and size, 


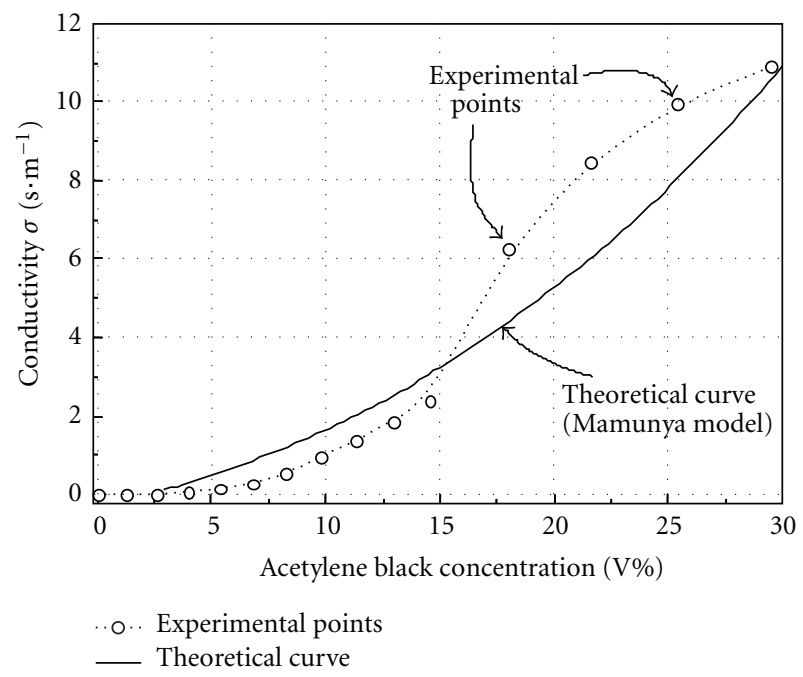

Figure 6: Variation of the theoretical (Mamunya model) and the experimental conductivity as a function of acetylene black concentration in the polypropylene-based composites.

TABLE 1: Theoretical parameters of the Mamunya model.

\begin{tabular}{lcc}
\hline & \multicolumn{3}{c}{ Composite } \\
Parameters & $\mathrm{PP} / \mathrm{CB}$ & $\mathrm{PP} / \mathrm{AB}$ \\
\hline$\sigma_{c}\left(\mathrm{~S} \cdot \mathrm{m}^{-1}\right)$ & 0.014 & 0.0127 \\
$\sigma_{F}\left(\mathrm{~S} \cdot \mathrm{m}^{-1}\right)$ & 8.1 & 10.89 \\
$\phi_{c}(\mathrm{~V} \%)$ & 5.37 & 2.61 \\
$k$ & 0.2 & 0.25 \\
\hline
\end{tabular}

interaction parameters, formation of aggregates, processing techniques, and operating conditions (temperature, mixing time, shear forces, ...) can simultaneously affect the composite properties including the electrical conductivity. Indeed, some established laws have been changed in order to take into account the effect of these different parameters.

We also tried to establish an equation that models our experimental results with the mathematical models that already exist. We sought a model that could cover the evolution of the conductivity of the area of low concentration (conductivity near zero) up to the conductivity at maximum filler concentration. The mathematical model of Boltzmann [16] seems best suited to represent the change in the conductivity of our composites based on the filler concentration (4):

$$
y=A_{2}+\frac{A_{1}-A_{2}}{1+e^{\left(x-x_{0}\right) / d x}} .
$$

In our modelization, " $y$ " represents the theoretical conductivity $\left(y=\sigma_{\text {th }}\right)$. This model has been developed using the software Microcal Origin 6.0 [16] to achieve the functions applied to the composites studied. The experimental points agree well with the theoretical curves in all respects for both types of filler (Figure 7) and for both the linear scale or the semilogarithmic scale.

In the representation of the semilogarithmic scales with this model (after the percolation threshold), there is a better agreement of the theoretical curves (solid line) and the

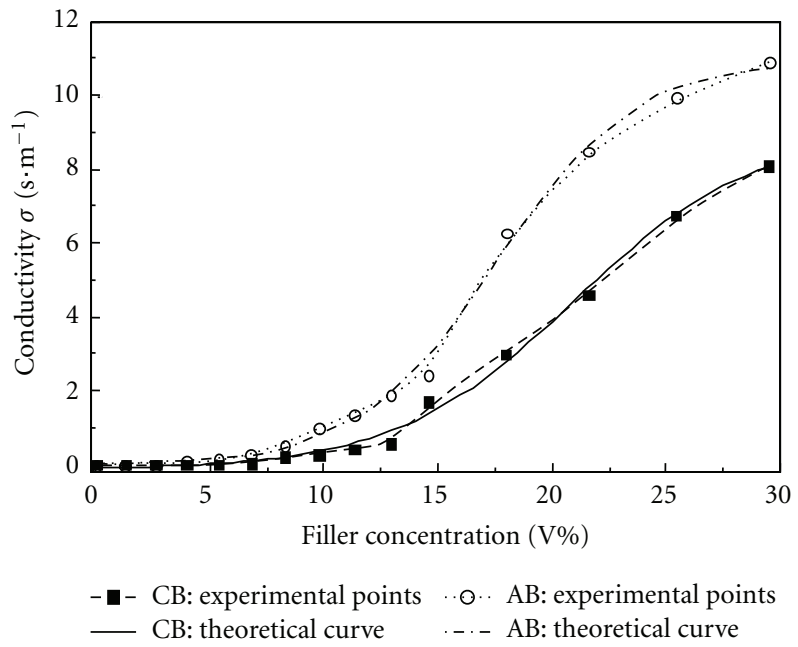

FIgURE 7: Comparative curves of the variation of the proposed model and the experimental conductivity as a function of carbon black and acetylene black fillers concentration in the polypropylenebased composites.

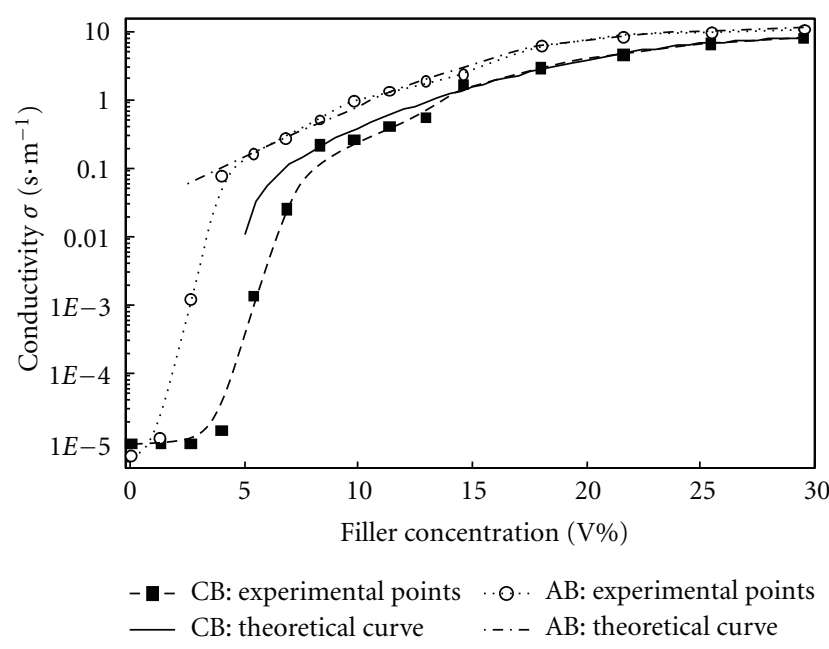

FIGURE 8: Semilogarithmic plot of the variation of the proposed model and the experimental conductivity as a function of carbon black and acetylene black fillers concentration in the polypropylenebased composites.

experimental points (dotted curve) for both types of fillers, than with Mamunya model (Figure 8).

(i) Boltzmann function is applied to the conductivity model of PP/CB composites:

$$
\sigma_{\text {th }}=9.015-\frac{9.16}{1+0.005 \cdot e^{x / 3.95}},
$$

where $x$ is the volume fraction of the filler concentration in the composite.

(ii) Boltzmann function applied to the conductivity model of $\mathrm{PP} / \mathrm{AB}$ composites:

$$
\sigma_{\text {th }}=10.92-\frac{10.92}{1+0.003 e^{x / 3}} .
$$




\section{Conclusion}

The dispersion of conductive particles in an insulation organic matrix imparts to the composites an electrical conductivity, which increases with the volume fraction of particles above a specific value (percolation threshold). This is an insulator/conductor transition, which can be described by the percolation theories.

There are many factors that affect the properties of polymeric composites, so it is very difficult to establish theoretical mathematical models that can be used to predict with a great extent of precision the properties of the developed material.

The results of the electrical characterization (conductivity) of polypropylene composites with carbon black or acetylene black confirm the existence of a percolation threshold, which is located at a lower filler concentration in the case of composites of the acetylene black.

At equal filler concentration, $\mathrm{PP} / \mathrm{AB}$ composites show a better conductivity than $\mathrm{PP} / \mathrm{CB}$ composites.

The filler type and size affect considerably the percolation locations.

The theoretical models established in order to predict the behavior of such composites, such as that of Mamunya are relatively in a better agreement with our experimental results.

The representation on a semilogarithmic scale, used with Mamunya model, can better detect the percolation threshold in composites, whereas in the representation in linear scales, significant uncertainties may affect the location of the percolation threshold.

The models we have developed from the Boltzmann equation cover the conductivity prediction of the prepared composites throughout the entire concentration range studied in linear representation of the results.

These models are more efficient and more accurate than the Mamunya model (semilogarithmic scale) and are in perfect agreement with the experimental results, for the two types of fillers. A better superposition of the two curves is observed, for both two types of fillers, than with Mamunya model.

\section{References}

[1] A. Boudenne, L. Ibos, M. Fois, J. C. Majesté, and E. Géhin, "Electrical and thermal behavior of polypropylene filled with copper particles," Composites Part A, vol. 36, no. 11, pp. 15451554, 2005.

[2] B. C. Muñoz, G. Steinthal, and S. Sunshine, "Conductive polymer-carbon black composites-based sensor arrays for use in an electronic nose," Sensor Review, vol. 19, no. 4, pp. 300305, 1999.

[3] X. M. Dong, R. W. Fu, M. Q. Zhang, B. Zhang, J. R. Li, and M. Z. Rong, "A novel sensor for organic solvent vapors based on conductive amorphous polymer composites: carbon black/poly(butyl methacrylate)," Polymer Bulletin, vol. 50, no. 1-2, pp. 99-106, 2003.

[4] D. L. Chinaglia, G. Gozzi, T. F. Schmidt et al., "Fabrication of novel light-emitting devices based on green-phosphor/ conductive-polymer composites," Philosophical Magazine Letters, vol. 87, no. 6, pp. 403-408, 2007.
[5] W. Zhang, A. A. Dehghani-Sanij, and R. S. Blackburn, "Carbon based conductive polymer composites," Journal of Materials Science, vol. 42, no. 10, pp. 3408-3418, 2007.

[6] F. Lux, "Models proposed to explain the electrical conductivity of mixtures made of conductive and insulating materials," Journal of Materials Science, vol. 28, no. 2, pp. 285-301, 1993.

[7] S. Kirkpatrick, "Percolation and Conduction," Reviews of Modern Physics, vol. 45, no. 4, pp. 574-588, 1973.

[8] D. S. McLachlan, M. Blaszkiewicz, and R. E. Newnham, "Electrical resistivity of composites," Journal of the American Ceramic Society, vol. 73, no. 8, pp. 2187-2203, 1990.

[9] K. Miyasaka, K. Watanabe, E. Jojima, H. Aida, M. Sumita, and K. Ishikawa, "Electrical conductivity of carbon-polymer composites as a function of carbon content," Journal of Materials Science, vol. 17, no. 6, pp. 1610-1616, 1982.

[10] R. D. Sherman, L. M. Middleman, and S. M. Jacobs, "Electron transport processes in conductor-filled polymers," Polymer Engineering and Science, vol. 23, no. 1, pp. 36-46, 1983.

[11] M. T. Clarkson, "Electrical conductivity and permittivity measurements near the percolation transition in a microemulsion. II. Interpretation," Physical Review A, vol. 37, no. 6, pp. 20792090, 1988.

[12] M. T. Kortschot and R. T. Woodhams, "Computer simulation of the electrical conductivity of polymer composites containing metallic fillers," Polymer Composites, vol. 9, no. 1, pp. 6071, 1988.

[13] E. P. Mamunya, V. V. Davidenko, and E. V. Lebedev, Dopovidi Akademii Nauk Ukrainskoi RSR, vol. 5, p. 124, 1991.

[14] E. P. Mamunya, V. V. Davidenko, and E. V. Lebedev, "Effect of polymer-filler interface interactions on percolation conductivity of thermoplastics filled with carbon black," Composite Interfaces, vol. 4, no. 4, pp. 169-176, 1997.

[15] M. L. Clingerman, J. A. King, K. H. Schulz, and J. D. Meyers, "Evaluation of electrical conductivity models for conductive polymer composites," Journal of Applied Polymer Science, vol. 83, no. 6, pp. 1341-1356, 2002.

[16] Microcal softwar Inc, Microcal Origin Version 6.0 [CDROM], Microcal softwar Inc, Northampton, Mass, USA, 1991.

[17] Z. M. Elimat, A. M. Zihlif, and G. Ragosta, "DC electrical conductivity of poly(methyl methacrylate)/carbon black composites at low temperatures," Journal of Materials Science, vol. 19, no. 11, pp. 1035-1038, 2008.

[18] F. Deng and Q.-S. Zheng, "An analytical model of effective electrical conductivity of carbon nanotube composites," Applied Physics Letters, vol. 92, no. 7, Article ID 071902, 2008.

[19] J. C. Huang, "Carbon black filled conducting polymers and polymer blends," Advances in Polymer Technology, vol. 21, no. 4, pp. 299-313, 2002.

[20] A. V. Goncharenko and E. F. Venger, "Percolation threshold for Bruggeman composites," Physical Review E, vol. 70, no. 5, Article ID 057102, 2004.

[21] Y. P. Mamunya, V. V. Davydenko, P. Pissis, and E. V. Lebedev, "Electrical and thermal conductivity of polymers filled with metal powders," European Polymer Journal, vol. 38, no. 9, pp. 1887-1897, 2002.

[22] E. P. Mamunya, V. V. Davidenko, and E. V. Lebedev, "Percolation conductivity of polymer composites filled with dispersed conductive filler," Polymer Composites, vol. 16, no. 4, pp. 319324, 1995.

[23] M. L. Clingerman, E. H. Weber, J. A. King, and K. H. Schulz, "Development of an additive equation for predicting the electrical conductivity of carbon-filled composites," Journal of Applied Polymer Science, vol. 88, no. 9, pp. 2280-2299, 2003. 
[24] J. M. Keith, J. A. King, and B. A. Johnson, "Electrical conductivity modeling of carbon filled polypropylene based resins for fuel cell bipolar plate applications," Journal of New Materials for Electrochemical Systems, vol. 11, no. 4, pp. 253-257, 2008.

[25] D. M. Bigg, "An investigation of the effect of carbon black structure, polymer morphology, and processing history on the electrical conductivity of carbon black filled thermoplastics," Journal of Rheology, vol. 28, no. 5, pp. 501-516, 1984.

[26] J. C. Dubois, "Propriétés diélectriques," Techniques de l'ingénieur, vol. 3140, no. 1, p. 4, 2004. 

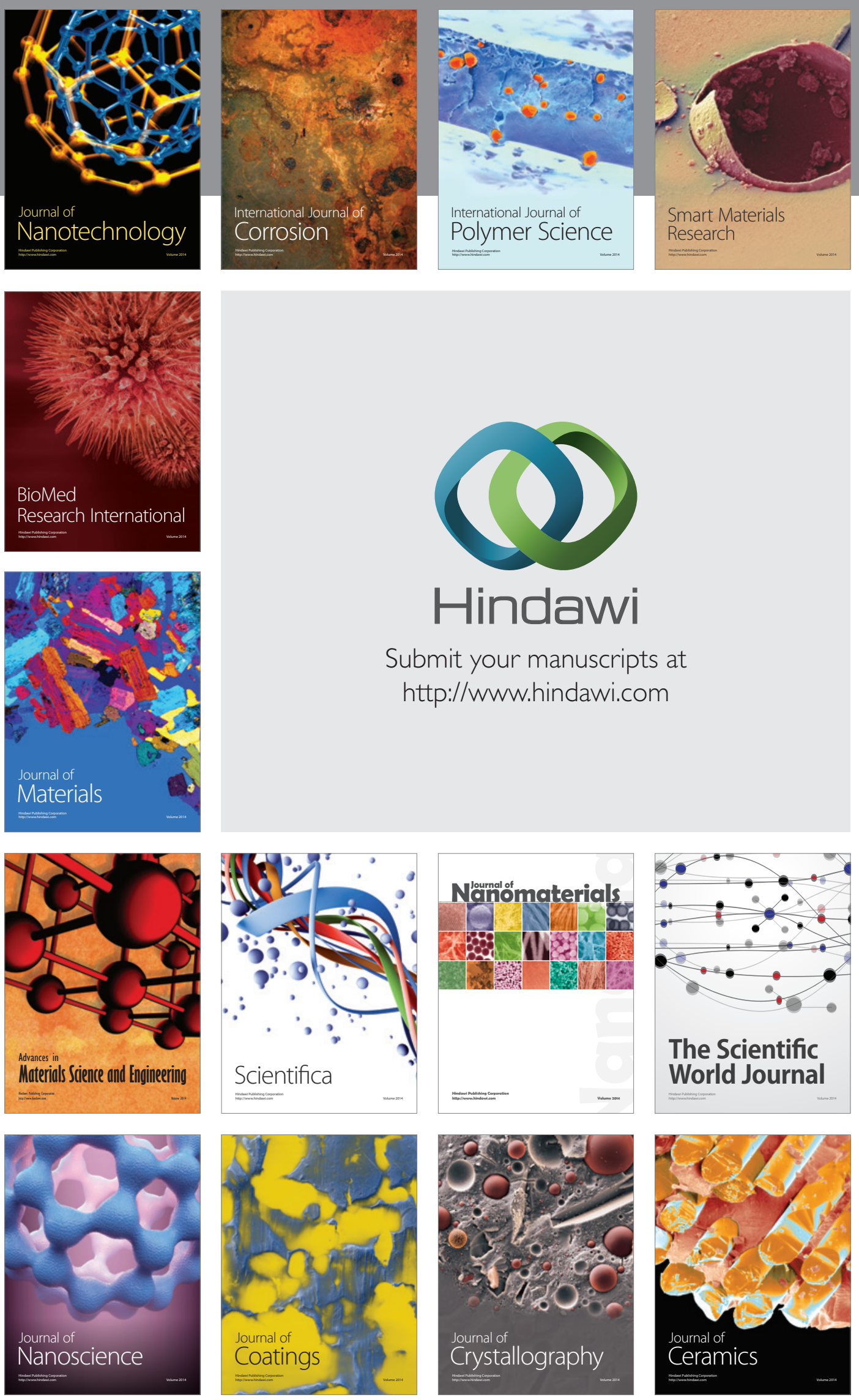

The Scientific World Journal

Submit your manuscripts at

http://www.hindawi.com

\section{World Journal}

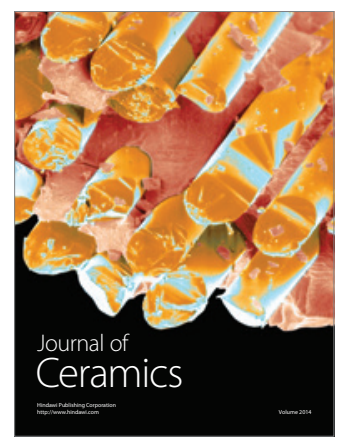

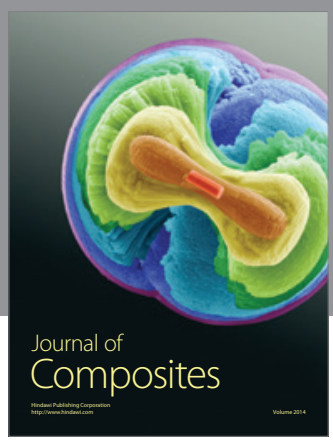
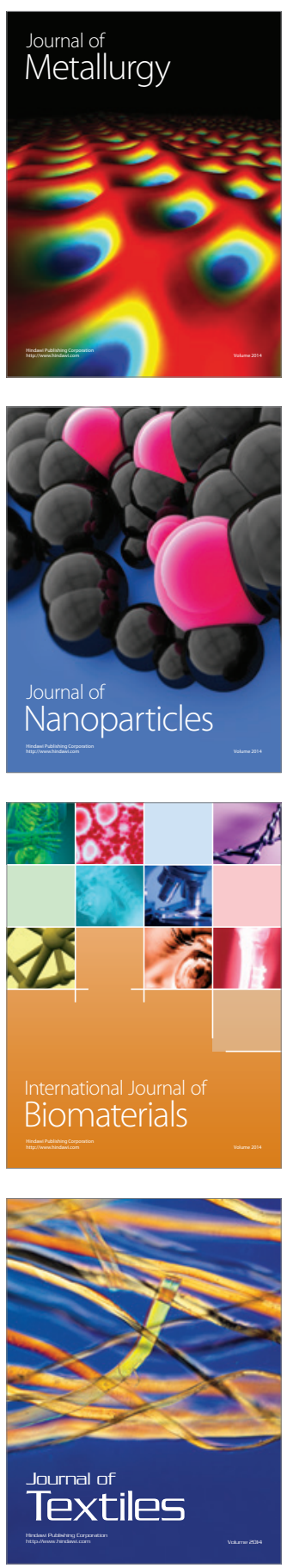